\title{
Revisiting Defenses against Large-Scale Online Password Guessing Attacks
}

\author{
Mansour Alsaleh, Mohammad Mannan, and P.C. van Oorschot, Member, IEEE
}

\begin{abstract}
Brute force and dictionary attacks on password-only remote login services are now widespread and ever increasing. Enabling convenient login for legitimate users while preventing such attacks is a difficult problem. Automated Turing Tests (ATTs) continue to be an effective, easy-to-deploy approach to identify automated malicious login attempts with reasonable cost of inconvenience to users. In this paper, we discuss the inadequacy of existing and proposed login protocols designed to address largescale online dictionary attacks (e.g., from a botnet of hundreds of thousands of nodes). We propose a new Password Guessing Resistant Protocol (PGRP), derived upon revisiting prior proposals designed to restrict such attacks. While PGRP limits the total number of login attempts from unknown remote hosts to as low as a single attempt per username, legitimate users in most cases (e.g., when attempts are made from known, frequently-used machines) can make several failed login attempts before being challenged with an ATT. We analyze the performance of PGRP with two real-world data sets and find it more promising than existing proposals.
\end{abstract}

Index Terms-Online password guessing attacks, brute force attacks, password dictionary, ATTs.

\section{INTRODUCTION}

$\mathrm{O}$ NLINE guessing attacks on password-based systems are inevitable and commonly observed against web applications and SSH logins. In a recent report, SANS [20] identified password guessing attacks on websites as a top cyber security risk. As an example of SSH passwordguessing attacks, one experimental Linux honeypot setup has been reported [18] to suffer on average 2,805 SSH malicious login attempts per computer per day (see also [8]). Interestingly, SSH servers that disallow standard password authentication may also suffer guessing attacks, e.g., through the exploitation of a lesser known/used SSH server configuration called keyboard interactive authentication [19]. However, online attacks have some inherent disadvantages compared to offline attacks: attacking machines must engage in an interactive protocol, thus allowing easier detection; and in most cases, attackers can try only limited number of guesses from a single machine before being locked out, delayed, or challenged to answer Automated Turing Tests (ATTs, e.g., CAPTCHAs [24]). Consequently, attackers often must employ a large number of machines to avoid detection or lock-out. On the other hand, as users generally choose common and relatively weak passwords (thus allowing effective password dictionaries [13], [25]), and attackers currently control large botnets (e.g., Conficker [15]), online attacks are much easier than before.

- M. Alsaleh and P.C. van Oorschot are with the School of Computer Science, Carleton University, 5145 Herzberg building, 1125 Colonel By Drive, Ottawa, ON K1S 5B6, Canada.

E-mail:manssour@gmail.com,paulv@scs.carleton.ca.

- M. Mannan is with the Concordia Institute for Information Systems Engineering, Concordia University, 1515 Ste-Catherine Street West, EV7.640, Montreal, QC, H3G 2W1 Canada.

E-mail:mmannan@ciise.concordia.ca.

Manuscript received 7 Sept. 2010; revised 17 Feb. 2011; accepted 22 Mar. 2011; published online 27 Apr. 2011.

For information on obtaining reprints of this article, please send e-mail to: tdsc@computer.org, and reference IEEECS Log Number TDSC-2010-09-0153. Digital Object Identifier no. 10.1109/TDSC.2011.24.
One effective defense against automated online password guessing attacks is to restrict the number of failed trials without ATTs to a very small number (e.g., three), limiting automated programs (or bots) as used by attackers to three free password guesses for a targeted account, even if different machines from a botnet are used. However, this inconveniences the legitimate user who then must answer an ATT on the next login attempt.

Several other techniques are deployed in practice, including: allowing login attempts without ATTs from a different machine, when a certain number of failed attempts occur from a given machine; allowing more attempts without ATTs after a time-out period; and time-limited account locking. Many existing techniques and proposals involve ATTs, with the underlying assumption that these challenges are sufficiently difficult for bots and easy for most people. However, users increasingly dislike ATTs as these are perceived as an (unnecessary) extra step; see Yan and Ahmad [28] for usability issues related to commonly used CAPTCHAs. Due to successful attacks which break ATTs without human solvers (e.g., [27], [22]), ATTs perceived to be more difficult for bots are being deployed. As a consequence of this arms-race, present-day ATTs are becoming increasingly difficult for human users [3], fueling a growing tension between security and usability of ATTs. Therefore, we focus on reducing user annoyance by challenging users with fewer ATTs, while at the same time subjecting bot logins to more ATTs, to drive up the economic cost to attackers [11].

Two well-known proposals for limiting online guessing attacks using ATTs are Pinkas and Sander [17] (herein denoted PS), and van Oorschot and Stubblebine [23] (herein denoted VS). For convenience, a review of these protocols is given in Section 6. The PS proposal reduces the number of ATTs sent to legitimate users, but at some meaningful loss of security; for example, in an example setup (with $p=0.05$, the fraction of incorrect login attempts requiring an ATT) PS allows attackers to eliminate 95 percent of the password space without answering any ATTs. The VS proposal 
reduces this but at a significant cost to usability; for example, VS may require all users to answer ATTs in certain circumstances (see Section 6). The proposal in the present paper, called Password Guessing Resistant Protocol (PGRP), significantly improves the security-usability trade-off, and can be more generally deployed beyond browser-based authentication.

PGRP builds on these two previous proposals. In particular, to limit attackers in control of a large botnet (e.g., comprising hundreds of thousands of bots), PGRP enforces ATTs after a few (e.g., three) failed login attempts are made from unknown machines. On the other hand, PGRP allows a high number (e.g., 30) of failed attempts from known machines without answering any ATTs. We define known machines as those from which a successful login has occurred within a fixed period of time. These are identified by their IP addresses saved on the login server as a white list, or (as in PS [17]) cookies stored on client machines. A white-listed IP address and/or client cookie expire after a certain time.

PGRP accommodates both graphical user interfaces (e.g., browser-based logins) and character-based interfaces (e.g., SSH logins), while the previous protocols deal exclusively with the former, requiring the use of browser cookies. PGRP uses either cookies or IP addresses, or both for tracking legitimate users. Tracking users through their IP addresses also allows PGRP to increase the number of ATTs for password guessing attacks and meanwhile to decrease the number of ATTs for legitimate login attempts. Although NATs and web proxies may (slightly) reduce the utility of IP address information, in practice, the use of IP addresses for client identification appears feasible [4]. In recent years, the trend of logging in to online accounts through multiple personal devices (e.g., PCs, laptops, smart phones) is growing. When used from a home environment, these devices often share a single public IP address (i.e., a simple NAT address) which makes IP-based history tracking more user friendly than cookies. For example, cookies must be stored, albeit transparently to the user, in all devices used for login.

\subsection{Contributions}

1. STRICT BUT USER-FRIENDLY ATT-BASED SCHEME. The proposed PGRP scheme is more restrictive against attackers than commonly used countermeasures and two earlier proposals [17], [23]. At the same time, PGRP requires answering fewer ATTs for all legitimate users, including those who occasionally require multiple attempts to recall a password.

2. FIRST REPORTED EMPIRICAL ANALYSIS OF ATTBASED SCHEMES. We compare PGRP's performance and usability (e.g., the number of ATTs triggered, ATTs sent to legitimate users) to previous such schemes, using two data sets from a university environment (SSH and web-email login data, covering more than a year).

3. APPLICABILITY TO WEB AND TEXT LOGINS. PGRP is not limited to web only login (unlike proposals solely relying on browser cookies), as it uses IP address and/or other methods to identify a remote machine in addition to optionally using cookies. By using text-based ATTs (e.g., textcaptcha.com), SSH login can be adapted to use PGRP.

\subsection{Organization}

Section 2 discusses related work on prevention techniques for online dictionary attacks. Section 3 presents the PGRP login protocol. Section 4 compares PGRP with other ATT-based protocols in terms of security (Section 4.1), usability (Section 4.2), and required computational resources (Section 4.3). A summary of limitations comparing these protocols is given in Section 4.4. In Section 5, we evaluate PGRP and other ATTbased protocols on two different remote login data sets and analyze the results. Section 6 provides a review of the PS and VS protocols. Section 7 concludes.

\section{Related Work}

Although online password guessing attacks have been known since the early days of the Internet, there is little academic literature on prevention techniques. Account locking is a customary mechanism to prevent an adversary from attempting multiple passwords for a particular username. Although locking is generally temporary, the adversary can mount a DoS attack by making enough failed login attempts to lock a particular account. Delaying server response after receiving user credentials, whether the password is correct or incorrect, prevents the adversary from attempting a large number of passwords in a reasonable amount of time for a particular username. However, for adversaries with access to a large number of machines (e.g., a botnet), this mechanism is ineffective. Similarly, prevention techniques that rely on requesting the user machine to perform extra nontrivial computation prior to replying to the entered credentials are not effective with such adversaries.

As discussed in Section 1, ATT challenges are used in some login protocols to prevent automated programs from brute force and dictionary attacks. Pinkas and Sander [17] presented a login protocol (PS protocol) based on ATTs to protect against online password guessing attacks. It reduces the number of ATTs that legitimate users must correctly answer so that a user with a valid browser cookie (indicating that the user has previously logged in successfully) will rarely be prompted to answer an ATT. A deterministic function $(A s k A T T())$ of the entered user credentials is used to decide whether to ask the user an ATT. To improve the security of the PS protocol, van Oorschot and Stubblebine [23] suggested a modified protocol in which ATTs are always required once the number of failed login attempts for a particular username exceeds a threshold; other modifications were introduced to reduce the effects of cookie theft.

For both PS and VS protocols, the decision function AskATT() requires careful design. He and Han [9] pointed out that a poor design of this function may make the login protocol vulnerable to attacks such as the "known function attack" (e.g., if a simple cryptographic hash function of the username and the password is used as $\operatorname{AskATT())}$ and "changed password attack" (i.e., an adversary mounts a dictionary attack before and after a password change event initiated by a valid user). The authors proposed a secure nondeterministic keyed hash function as $A \operatorname{skATT}()$ so that each username is associated with one key that should be changed whenever the corresponding password is changed. The proposed function requires extra server-side storage per username and at least one cryptographic hash operation per login attempt. 
Input:

$t_{1}(\mathrm{def}=30 d), t_{2}(\mathrm{def}=1 d), t_{3}(\mathrm{def}=1 d), k_{1}(\mathrm{def}=30), k_{2}(\mathrm{def}=3)$

// The keyword 'def' denotes the default parameter value and ' $d$ ' denotes day, $k_{1}, k_{2} \geq 0$

// For an explanation of the use of expiry intervals, see Section 3.2 under 'Data structures' un, pw, cookie

//username, password, and remote host's browser cookie if any

//white list of IP addresses with successful login

$F T$ (global variable, def $=0$, expires after $t_{2}$ ) $/ /$ table of number of failed logins per username

$F S$ (global variable, def=0, expires after $t_{3}$ ) //table of number of failed logins indexed by (srcIP, username) for hosts in $w$ or hosts with valid cookies

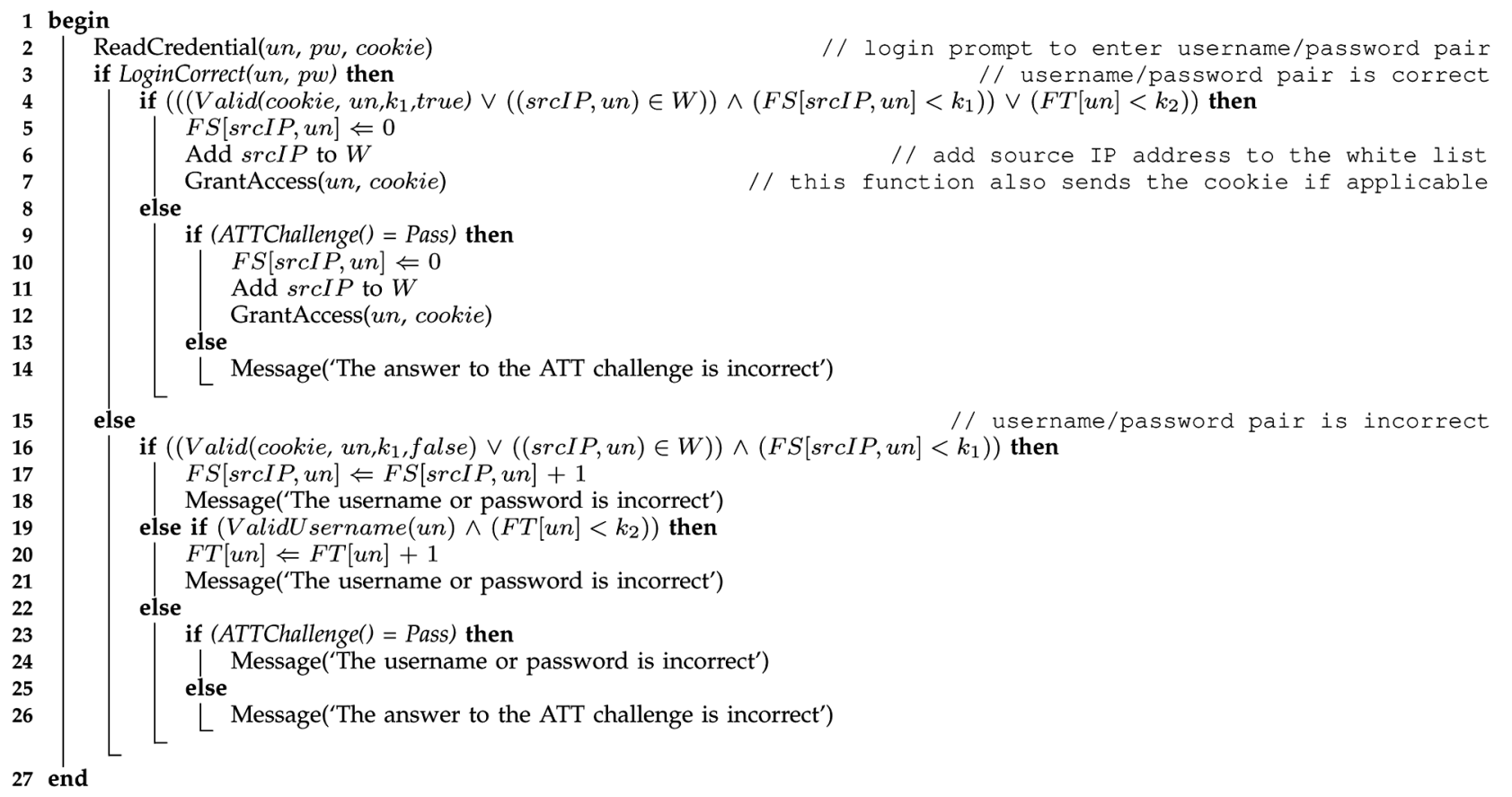

Fig. 1. PGRP: Password Guessing Resistant Protocol.

\section{Password Guessing Resistant Protocol}

In this section, we present the PGRP protocol, including the goals and design choices.

\subsection{Goals, Operational Assumptions and Overview}

\subsubsection{Protocol Goals}

Our objectives for PGRP include the following:

1. The login protocol should make brute force and dictionary attacks ineffective even for adversaries with access to large botnets (i.e., capable of launching the attack from many remote hosts).

2. The protocol should not have any significant impact on usability (user convenience). For example: for legitimate users, any additional steps besides entering login credentials should be minimal. Increasing the security of the protocol must have minimal effect in decreasing the login usability.

3. The protocol should be easy to deploy and scalable, requiring minimum computational resources in terms of memory, processing time, and disk space.

\subsubsection{Assumptions}

We assume that adversaries can solve a small percentage of ATTs, e.g., through automated programs, brute force mechanisms, and low paid workers (e.g., Amazon Mechanical Turk [1]). Incidents of attackers using IP addresses of known machines and cookie theft for targeted password guessing are also assumed to be minimal. Traditional password-based authentication is not suitable for any untrusted environment (e.g., a keylogger may record all keystrokes, including passwords in a system, and forward those to a remote attacker). We do not prevent existing such attacks in untrusted environments, and thus essentially assume any machines that legitimate users use for login are trustworthy. The data integrity of cookies must be protected (e.g., by a MAC using a key known only to the login server [17]).

\subsubsection{Overview}

The general idea behind PGRP (see Fig. 1) is that except for the following two cases, all remote hosts must correctly answer an ATT challenge prior to being informed whether access is granted or the login attempt is unsuccessful: 1) when the number of failed login attempts for a given username is very small; and 2) when the remote host has successfully logged in using the same username in the past (however, such a host must pass an ATT challenge if it generates more failed login attempts than a prespecified threshold).

In contrast to previous protocols, PGRP uses either IP addresses, cookies, or both to identify machines from which users have been successfully authenticated. The decision to require an ATT challenge upon receiving incorrect credentials is based on the received cookie (if any) and/or the remote host's IP address. In addition, if the number of failed login attempts for a specific username is below a threshold, the user is not required to answer an 
ATT challenge even if the login attempt is from a new machine for the first time (whether the provided usernamepassword pair is correct or incorrect). Section 3.3 below discusses these differences in further detail.

\subsection{Data Structure and Function Description}

\subsubsection{Data Structures}

PGRP maintains three data structures:

1. $W$. A list of \{source IP address, username\} pairs such that for each pair, a successful login from the source IP address has been initiated for the username previously.

2. FT. Each entry in this table represents the number of failed login attempts for a valid username, un. A maximum of $k_{2}$ failed login attempts are recorded. Accessing a nonexisting index returns 0 .

3. FS. Each entry in this table represents the number of failed login attempts for each pair of $(\operatorname{srcIP}$, un). Here, $\operatorname{srcIP}$ is the IP address for a host in $W$ or a host with a valid cookie, and $u n$ is a valid username attempted from $\operatorname{srcIP}$. A maximum of $k_{1}$ failed login attempts are recorded; crossing this threshold may mandate passing an ATT (e.g., depending on $F T[u n])$. An entry is set to 0 after a successful login attempt. Accessing a nonexisting index returns 0 .

Each entry in $W, F T$, and $F S$ has a "write-expiry" interval such that the entry is deleted when the given period of time $\left(t_{1}, t_{2}\right.$, or $\left.t_{3}\right)$ has lapsed since the last time the entry was inserted or modified. There are different ways to implement write-expiry intervals (e.g., hashbelt [14]). A simple approach is to store a timestamp of the insertion time with each entry such that the timestamp is updated whenever the entry is modified. At anytime the entry is accessed, if the delta between the access time and the entry timestamp is greater than the data structure write-expiry interval (i.e., $t_{1}, t_{2}$, or $t_{3}$ ), the entry is deleted.

\subsubsection{Functions}

PGRP uses the following functions (IN denotes input and OUT denotes output):

1. ReadCredential (OUT: un, pw, cookie). Shows a login prompt to the user and returns the entered username and password, and the cookie received from the user's browser (if any).

2. LoginCorrect (IN : un, pw; OUT: true/false). If the provided username-password pair is valid, the function returns true; otherwise, it returns false.

3. GrantAccess (IN: un, cookie). The function sends the cookie to the user's browser and then enables access to the specified user account.

4. Message (IN: text). Shows a text message.

5. ATTChallenge (OUT: Pass/Fail). Challenges the user with an ATT and returns "Pass" if the answer is correct; otherwise, it returns "Fail."

6. ValidUsername (IN: un; OUT: true/false). If the provided username exists in the login system, the function returns true; otherwise, it returns false.

7. Valid(IN: cookie, un, $k_{1}$, state; OUT: cookie,true/false). First, the function checks the validity of the cookie (if any) where it is considered invalid in the following cases: 1) the login username does not match the cookie username; 2) the cookie is expired; or 3) the cookie counter is equal to or greater than $k_{1}$. The function returns true only when a valid cookie is received. If state $=$ true (i.e., the entered user credentials are correct, as in line 4 of Fig. 1), a new cookie is created (if cookies are supported in the login system) including the following information: username, expiry date, and a counter of the number of failed login attempts (since the last successful login; initialized to 0 ). Notice that if state $=$ true, the function does not send the created cookie to the user's browser. Rather, the cookie is sent later by the GrantAccess() function. If state = false (i.e., the entered user credentials are incorrect, as in line 16 of Fig. 1) and a valid cookie is received, the cookie counter is incremented by one and the cookie is sent back to the user's browser. No action is performed for all the other cases.

\subsection{Cookies versus Source IP Addresses}

Similar to the previous protocols, PGRP keeps track of user machines from which successful logins have been initiated previously. Browser cookies seem a good choice for this purpose if the login server offers a web-based interface. Typically, if no cookie is sent by the user browser to the login server, the server sends a cookie to the browser after a successful login to identify the user on the next login attempt. However, if the user uses multiple browsers or more than one OS on the same machine, the login server will be unable to identify the user in all cases. Cookies may also be deleted by users, or automatically as enabled by the private browsing mode of most modern browsers. Moreover, cookie theft (e.g., through session hijacking) might enable an adversary to impersonate a user who has been successfully authenticated in the past [7]. In addition, using cookies requires a browser interface (which, e.g., is not applicable to SSH).

Alternatively, a user machine can be identified by the source IP address. Relying on source IP addresses to trace users may result in inaccurate identification for various reasons, including: 1) the same machine might be assigned different IP addresses over time (e.g., through the network DHCP server and dial-up Internet); and 2) a group of machines might be represented by a smaller number or even a single Internet-addressable IP address if a NAT mechanism is in place. However, most NATs serve few hosts and DHCPs usually rotate IP addresses on the order of several days [4] (also, techniques to identify machines behind a NAT exist, e.g., [2], [10]).

Drawbacks of identifying a user by means of either a browser cookie or a source IP address include: 1) failing to identify a machine from which the user has authenticated successfully in the past; and 2) wrongly identifying a machine the user has not authenticated before. Case 1) decreases usability since the user might be asked to answer an ATT challenge for both correct and incorrect login credentials. On the other hand, case 2) affects security since some users / attackers may not be asked to answer an ATT challenge even though they have not logged in successfully from those machines in the past. However, the probability of launching a dictionary or brute force attack from these machines appears to be low. First, for identification through cookies, a directed attack to steal users' cookies is required by an adversary. 
Second, for identification through IP addresses, the adversary must have access to a machine in the same subnet as the user.

Consequently, we choose to use both browser cookies and source IP address (or only one of them if the other is not applicable) in PGRP to minimize user inconvenience during the login process. Also, by using IP addresses only, PGRP can be used in character-based login interfaces such as SSH. An SSH server can be adapted to use PGRP using text-based ATTs (e.g., textcaptcha.com). For example, a prototype of a text-based CAPTCHA for SSH is available as a source code patch for OpenSSH [12].

The security implications of mistakenly treating a machine as one that a user has previously successfully logged in from is limited by a threshold such that after a specific number of failed login attempts ( $k_{1}$ in Fig. 1), an ATT challenge is imposed. For identification through a source IP address, the condition $F S[\operatorname{srcIP}$, un $]<k_{1}$ in line 4 (for correct credentials) and in line 16 (for incorrect credentials) limits the number of failed login attempts an identified user can make without answering ATTs (see Fig. 1). Also, as explained in Section 3.2, the function Valid (cookie, un, $k_{1}$, true) in line 4 updates a counter in the received cookie in which the cookie is considered invalid once this counter hits or exceeds $k_{1}$. This function is also called in line 16 to check this counter in case of a failed login attempt.

\subsection{Decision Function for Requesting ATTs}

Below we discuss issues related to ATT challenges as provided by the login server in Fig. 1. The decision to challenge the user with an ATT depends on two factors: 1) whether the user has authenticated successfully from the same machine previously; and 2) the total number of failed login attempts for a specific user account. For definitions of $W, F T$, and $F S$, see Section 3.2.

\subsubsection{Username-Password Pair Is Valid}

As in the condition in line 4, upon entering a correct username-password pair, the user will not be asked to answer an ATT challenge in the following cases:

1. A valid cookie is received from the user machine (i.e., the function Valid returns true) and the number of failed login attempts from the user machine's IP address for that username, $F S[\operatorname{srcIP}, u n]$, is less than $k_{1}$ over a time period determined by $t_{3}$;

2. The user machine's IP address is in the whitelist $W$ and the number of failed login attempts from this IP address for that username, $F S[\operatorname{srcIP}$, un], is less than $k_{1}$ over a time period determined by $t_{3}$;

3. The number of failed login attempts from any machine for that username, $F T[u n]$, is below a threshold $k_{2}$ over a time period determined by $t_{2}$.

The last case enables a user who tries to login from a new machine/IP address for the first time before $k_{2}$ is reached to proceed without an ATT. However, if the number of failed login attempts for the username exceeds the threshold $k_{2}$ (default 3 ), this might indicate a guessing attack and hence the user must pass an ATT challenge.

\subsubsection{Username-Password Pair Is Invalid}

Upon entering an incorrect username-password pair, the user will not be asked to answer an ATT challenge in the following cases:
1. A valid cookie is received from the user machine (i.e., the function Valid returns true) and the number of failed login attempts from the user machine's IP address for that username, $F S[\operatorname{srcIP}, u n]$, is less than $k_{1}$ (line 16) over a time period determined by $t_{3}$;

2. The user machine's IP address is in the whitelist $W$ and the number of failed login attempts from this IP address for that username, $F S[\operatorname{srcIP}, u n]$, is less than $k_{1}$ (line 16) over a time period determined by $t_{3}$;

3. The username is valid and the number of failed login attempts (from any machine) for that username, $F T[u n]$, is below a threshold $k_{2}$ (line 19) over a time period determined by $t_{2}$.

A failed login attempt from a user with a valid cookie or in the whitelist $W$ will not increase the total number of failed login attempts in the $F T$ table since it is expected that legitimate users may potentially forget or mistype their password (line 16-18). Nevertheless, if the user machine is identified by a cookie, a corresponding counter of the failed login attempts in the cookie will be updated. In addition, the $F S$ entry indexed by the \{source IP address, username\} pair will also be incremented (line 17). Once the cookie counter or the corresponding $F S$ entry hits or exceeds the threshold $k_{1}$ (default value 30 ), the user must correctly answer an ATT challenge.

\subsubsection{Output Messages}

PGRP shows different messages in case of incorrect \{username, password\} pair (lines 21 and 24) and incorrect answer to the given ATT challenge (lines 14 and 26). While showing a human that the entered \{username, password\} pair is incorrect, an automated program unwilling to answer the ATT challenge cannot confirm whether it is the pair or the ATT that was incorrect. However, while this is more convenient for legitimate users, it gives more information to the attacker about the answered ATTs. PGRP can be modified to display only one message in lines 14,21 , 24 , and 26 (e.g., "login fails" as in the PS and VS protocols) to prevent such information leakage.

\subsubsection{Why Not to Black-List Offending IP Addresses}

We choose not to create a blacklist for IP addresses making many failed login attempts for the following reasons: 1 ) this list may consume considerable memory; 2 ) legitimate users from blacklisted IP addresses could be blocked (e.g., using compromised machines); and 3) hosts using dynamic IP addresses seem more attractive targets (compared to hosts with static IP addresses) for adversaries to launch their attacks from (e.g., spammers [26]).

If the cookie mechanism is not available for the login server, PGRP can operate by using only source IP addresses to keep track of user machines. Security and usability implications in this case are discussed in Section 4.

\section{Comparison With Other ATT-Based Protocols}

In this section, we analyze the security, usability, and required system resources of PGRP as compared to a strawman protocol and the PS and VS protocols (see Fig. 2, Fig. 3, and Fig. 4 in Section 6 for a review of these protocols). This section also provides a comparative summary of major limitations in each protocol. 


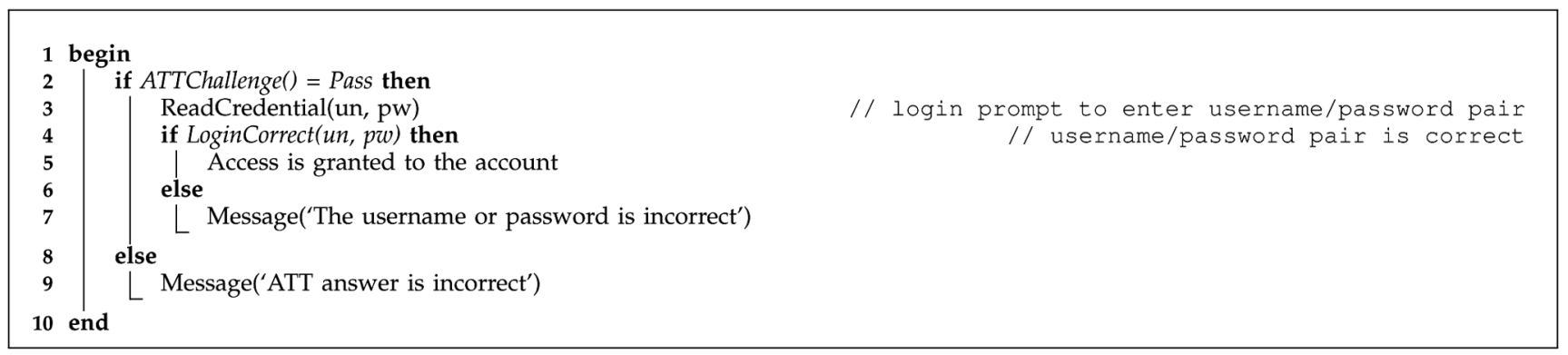

Fig. 2. Secure but inconvenient login protocol [17].

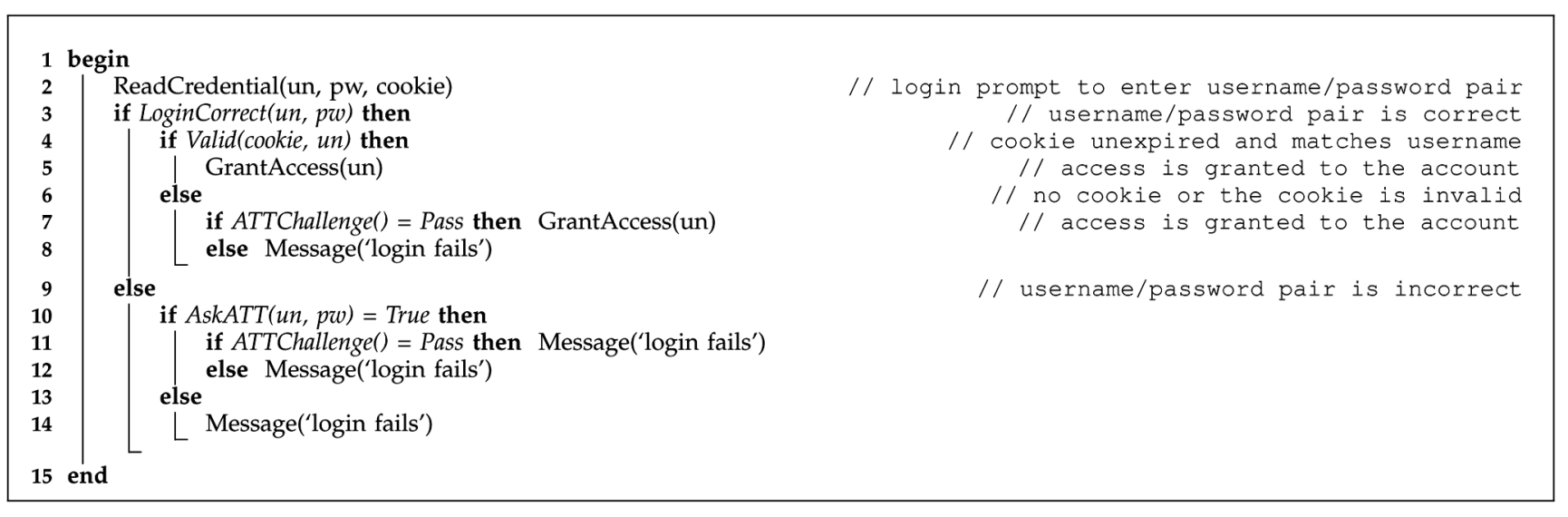

Fig. 3. PS protocol, adapted from Pinkas and Sander [17].

\subsection{Security Analysis}

Following the previous analysis of PS [17], assume a fixed password space of cardinality $N$, assume passwords are equi-probable, and that the delay between when the \{username, password\} pair is entered and the ATT challenge is presented to the user is identical whether or not the credentials are correct. Also assume that cookie theft, and adversaries using legitimate users' IP addresses ${ }^{1}$ occur rarely.

\subsubsection{Single-Account Attacks}

In a single account attack, a specific user account is targeted. Following the security analysis of VS [23] in this case, we consider the following questions:

- Q1. What is the expected number of passwords that an adversary can eliminate from the password space without answering any ATT challenge?

- Q2. What is the expected number of ATT challenges an adversary must answer to correctly guess a password?

- Q3. What is the probability of a confirmed correct guess for an adversary unwilling to answer any ATT?

- Q4. What is the probability of a confirmed correct guess for an adversary willing to answer $c$ ATTs?

Table 1 compares PGRP with the PS and VS protocols. For simplicity, we use only the case $c \geq 2$ in $\mathrm{Q} 4$ for the VS protocol. The answer to Q1 depends on the threshold $k_{2}$.

1. For example, in case of dynamic IP addresses, an attacker machine may be assigned an IP address previously used by a targeted user's machine.
The adversary can eliminate only $k_{2}$ passwords without answering ATTs. Likewise, for Q2, the expected number of ATTs the adversary must answer to correctly guess a password is one-half of the remaining passwords of the password space after subtracting the number of login attempts that do not require ATTs. Using a small value for $k_{2}$ yields $\frac{1}{2}\left(N-k_{2}\right) \approx \frac{1}{2} N$. For Q3, given that $k_{2}$ is intended to be small (e.g., 3), the probability of guessing a password for a single-account attack without answering any ATT is very small (e.g, for 8-char case-sensitive alphabetical passwords chosen randomly, $N=52^{8}$ and $p$ (guessing the right password) $=2 / 52^{8}$ ). For $\mathrm{Q} 4$, the adversary has only $k_{2}$ free attempts after which ATTs must be answered. Therefore, he can guess a total of $k_{2}+c$ passwords with a probability of $\left(k_{2}+c\right) / N$ to find a correct password.

This analysis shows that PGRP provides improved security over PS and VS with respect to all four questions, and identical security compared to the strawman protocol for $k_{2}=0$.

\subsubsection{Multiaccount Attacks}

In contrast to a directed attack on a single account, an adversary could attempt to break into multiple accounts at the same time. In fact, this is the current trend of brute force and dictionary attacks [20]. In this case, the adversary usually has access to a large number of machines (e.g., compromised machines in a botnet) and initiates the attack from many sources at the same time. This typically gives the adversary a greater chance of compromising user accounts than targeting a single account. 
Input:

$F T$ (global variable, def $=0$, expires after $t_{2}$ )//table of number of failed logins per username

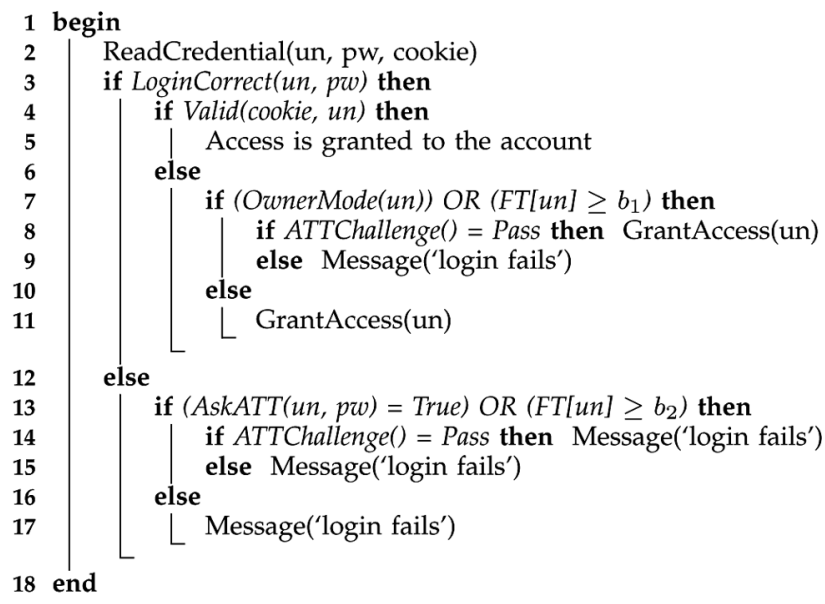

// login prompt to enter username/password pair $/ /$ username/password pair is correct // cookie unexpired and matches username

// no cookie or the cookie is invalid

$/ /$ access is granted to the account

$/ /$ access is granted to the account

// username/password pair is incorrect

Fig. 4. VS protocol, adapted from van Oorschot and Stubblebine [23]

TABLE 1

Comparative Security Analysis for Single-Account Attacks

\begin{tabular}{c|c|c|c|c|c|}
\cline { 2 - 5 } & Strawman & PS & \multicolumn{2}{|c|}{ VS [23] } & PGRP \\
\cline { 4 - 5 } & Protocol [17] & {$[17]$} & Owner & Non-owner & Protocol \\
\hline \hline Q1 & 0 & $N-p N$ & $(1-p) b_{2}$ & $\max \left(b_{1},(1-p) b_{2}\right)$ & $k_{2}$ \\
Q2 & $\frac{1}{2} N$ & $\frac{1}{2} p N$ & $\frac{1}{2}\left(N-(1-p) b_{2}\right) \approx \frac{1}{2} N$ & $\frac{1}{2}\left(N-\max \left(b_{1},(1-p) b_{2}\right)\right)$ & $\frac{1}{2}\left(N-k_{2}\right)$ \\
Q3 & 0 & 0 & 0 & $b_{1} / N$ & $k_{2} / N$ \\
Q4 & $c / N$ & $c / p N$ & $\leq \min \left(\frac{c}{p}, b_{2}+c\right) / N$ & $\left(b_{1}+c\right) / N$ & $\left(k_{2}+c\right) / N$ \\
\hline
\end{tabular}

Consider $k_{2}=3, p=0.05, b_{1}=5$, and $b_{2}=5$, for concreteness; see Section 6 for a review of the PS and VS algorithms.

We compare previous protocols and PGRP by answering the following questions in Table 2:

- Q1. What is the probability that an adversary knowing $m$ usernames can correctly guess a password without answering any ATT challenge?

- Q2. What is the probability of a confirmed correct guess for an adversary knowing $m$ usernames and willing to answer $c$ ATTs?

Considering Q1 in Table 2, PS appears more secure for multiaccount attacks than VS and PGRP. However, it may be unrealistic to assume that an adversary with access to a large botnet is unable to break a small percentage of ATTs [27], [22] (which leads us to Q2).

For Q2, the probability in PGRP depends on the number of usernames the adversary knows and $k_{2}$. While PGRP is comparable to the VS protocol in multiaccount attacks, PS seems slightly better than PGRP but only for login systems with a large number of users as in

$$
\begin{aligned}
\frac{m \cdot k_{2}+c}{N} & >\frac{c}{p \cdot N}, \\
m & >\frac{c}{k_{2}}\left(\frac{1}{p}-1\right) .
\end{aligned}
$$

To consider a concrete example, for any password length, $k_{2}=3, p=0.05$, and an adversary willing to answer $c=2^{20}$ ATTs: $m>1 / 3\left(2^{20} / 0.05-2^{20}\right)$; i.e., when $m>2^{22}$ users, PS is better than PGRP in Q2.
In the PGRP protocol, an adversary may be able to guess a subset of the valid usernames which is undesirable in certain cases [6]. In line 19 of Fig. 1, the FT list is not updated if the username is invalid, thus an ATT will be requested for each login attempt with an invalid username. Therefore, the adversary could generate a list of valid usernames as follows: if an attempted username requires an ATT for the first login attempt, the username is considered invalid; otherwise, the username is valid. However, the adversary will overlook valid usernames that have at least $k_{2}$ failed attempts. While the condition ValidUsername(un) in line 19 can be omitted to overcome this drawback, the number of entries in the list $F T$ will be now proportional to the number of all attempted usernames (whether valid or invalid) by users/attackers within a time period determined by $t_{2}$ (see Section 3.2 under "Data structures"). We choose to keep the condition ValidUsername(un) in line 19 to restrict the maximum size of $F T$ to the number of valid usernames, even when guessing attacks involving a large number of usernames (both valid and invalid) are launched.

\subsection{Usability Comments on ATT Challenges}

Our main security goal is to restrict an attacker who is in control of a large botnet from launching online singleaccount or multiaccount password dictionary attacks. In terms of usability, we want to reduce the number of ATTs sent to legitimate users as much as possible. A user receives ATTs when the total number of failed attempts exceeds 
TABLE 2

Comparative Security Analysis for Multiaccount Attacks

\begin{tabular}{c|c|c|c|c|c|}
\cline { 2 - 5 } & Strawman & PS & \multicolumn{2}{|c|}{ VS [23] } & PGRP \\
\cline { 4 - 5 } & Protocol [17] & [17] & Owner & Non-owner & Protocol \\
\hline \hline Q1 & 0 & 0 & 0 & $m b_{1} / N$ & $m k_{2} / N$ \\
Q2 & $c / N$ & $c / p N$ & $\leq m i n\left(\frac{c}{p}, b_{2}+c\right) / N$ & $\left(m b_{1}+c\right) / N$ & $\left(m k_{2}+c\right) / N$ \\
\hline
\end{tabular}

threshold $k_{2}$, and the login attempt is initiated from 1) an unknown machine (i.e., no valid cookies or white-listed IP addresses), or 2) a known machine from which the user has already failed $k_{1}$ times. This happens for both cases of correct and incorrect username-password pairs, assuming the provided username is valid. Below we discuss different login scenarios and the extra effort as required from users by PGRP. The analysis below indicates that only limited usability impact may be expected from our proposal; the same can also be inferred from our real-world data analysis, e.g., the number of ATTs sent to legitimate users (see Section 5). However, we have not yet carried out any formal user testing. For notation and parameters as used in the following, see Fig. 1. For definitions of $W, F T$, and $F S$, see "Data structures" in Section 3.2.

\subsubsection{First Time Login from an Unknown Machine}

If a valid username-password pair is provided from an unknown machine (i.e., one from which no successful login has occurred within a designated period), no ATTs are required if the total fail count from unknown machines is below $k_{2}$ (within a time period determined by $t_{2}$ ). This threshold may be exceeded as follows: 1) the user may provide incorrect passwords from that machine $k_{2}$ times; 2 ) attackers may have attempted $k_{2}$ failed passwords (from unknown machines); or 3) a combination of 1) and 2). Once a user successfully logs in, the machine's IP address is added to the known list $(W)$.

\subsubsection{Subsequent Login from a Known Machine}

ATTs are sent to a known machine (i.e., one from which a successful login has occurred within a designated period) only when $k_{1}$ is hit or crossed (see line 4 in Fig. 1) for that machine and the user account is possibly under attack (i.e., $k_{2}$ failed attempts also occurred on the account's username from unknown machines). By setting $k_{1}$ to be relatively large (e.g., $k_{1}=30$ ), legitimate users may make a reasonable number of password mistakes without experiencing any ATTs.

\subsubsection{Valid Password Is Provided}

Users may be understandably annoyed if they provide a valid password, and yet are asked to answer an ATT. When a valid password is provided by the user, no ATT challenges are sent if the attempt comes from a known machine which has not been used for more than $k_{1}-1$ failed login attempts within a time period determined by $t_{3}$. If the user hits or crosses the threshold $k_{1}$, still no ATTs are sent if the number of failed login attempts from unknown machines remains below $k_{2}$. Thus, users must pass ATT challenges only when they attempt login from unknown machines and the number of failed attempts from unknown machines has hit or crossed $k_{2}$ (possibly due to an ongoing attack). We believe this is an uncommon occurrence, as was apparent from our collected data.

\subsubsection{Invalid Password}

This may be a common occurrence for several reasons: 1) if users need multiple attempts to recall the correct password; 2) if users cycle-through multiple passwords due to multipassword interference [5]; and 3) typing errors including activating the caps lock key, sometimes aggravated by onscreen masking of password characters (see, e.g., Nielsen's blog [16]). From each known machine, a user is allowed up to $k_{1}$ attempts, before challenged with ATTs; i.e., if the user has logged in from $n$ known machines (within a time period determined by $\left.t_{3}\right)$, then in total $n \cdot k_{1}+k_{2}$ attempts are allowed without ATTs. While high values of $k_{1}$ (30 by default) provide convenient login for legitimate users in common use cases, we do not recommend very high values (e.g., $\left.k_{1}=10,000\right)$ as that may aid guessing attacks when a cookie is stolen or a dynamic white-listed IP address is assigned to an attacker's machine (i.e., a bot). Note that in VS [23], an adversary can make a certain number of failed connection attempts (the threshold $b_{2}$ in Fig. 4) for all (or as many as possible) users of system, with the result that any failed login attempt from a legitimate user will face an ATT challenge. In PGRP, user convenience is unaffected by an attacker's actions, as long as there are not more than $k_{1}-1$ unsuccessful login attempts from known machines.

\subsubsection{Invalid Username}

When a user tries login with a nonexistent username (e.g., typing errors), an ATT challenge is given. Irrespective of the password or ATT answer, the login fails. This feature restricts attackers from learning valid usernames (except the usernames obtained via brute force attacks as explained in Section 4.1.2), and improves protocol performance in terms of memory usage (i.e., no entries in protocol data structures $W, F T$, or $F S$ ). However, from a usability point of view, this is not ideal. We expect that this type of error would be limited in practice (in part because usernames, in contrast to passwords, are echoed on a display).

\subsection{System Resources}

No lists are maintained in the PS protocol (see Fig. 3), thus no extra memory overhead is imposed on the login server. In the VS protocol (see Fig. 4), only FT is maintained. The number of entries in this list grows linearly with unique usernames (both valid and invalid) used in failed login attempts. An attacker may try to exhaust a login server's memory by failed login attempts for many usernames. For any cookie-based login protocol, the login server may also need to store information regarding each generated cookie to ameliorate cookie theft attacks [23]. Note that neither the PS nor VS protocol uses IP addresses. The most expensive server operation in PS, VS, and PGRP is generating an ATT. 
TABLE 3

Comparison of Protocol Limitations (Limitations Are in Bold Face)

\begin{tabular}{|c|c|c|c|c|}
\hline & & PS [17] & VS [23] & PGRP \\
\hline \multirow{3}{*}{ 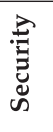 } & Passwords eliminated from the password space of cardinality $N$ & $(1-p) \mathbf{N}$ & $(\mathbf{1}-\mathbf{p}) \mathbf{b}_{2}$ & $k_{2}$ \\
\hline & Password space elimination by an adversary with a valid cookie & $\mathbf{N}$ & $\mathbf{N}$ & $k_{1}$ \\
\hline & Cookie theft & Yes & Yes & Yes \\
\hline \multirow{5}{*}{ 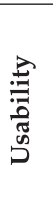 } & \multirow{2}{*}{ Probability of ATT for an incorrect password from known machine } & \multirow{2}{*}{$\mathbf{p}$} & \multirow{2}{*}{$\mathbf{p}$} & 0 (attempts $\left.<k_{1}\right)$ \\
\hline & & & & 1 (attempts $\left.\geq k_{1}\right)$ \\
\hline & Failed login attempts attack to force ATTs for legitimate users & No & Yes & No \\
\hline & ATTs for a correct password from unknown machine & Yes & In owner mode & if attempts $\geq k_{2}$ \\
\hline & Cookies drawbacks (multiple browsers/machines, deleted cookies) & Yes & Yes & No \\
\hline \multirow{4}{*}{ 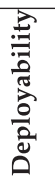 } & AskATT function required & Yes & Yes & No \\
\hline & Protocol is suitable for browsers only & Yes & Yes & No \\
\hline & Protocol state grows linearly with the number of users & No & Yes & Yes \\
\hline & Protocol state grows linearly with usernames in failed attempts & No & Yes & No \\
\hline
\end{tabular}

In PGRP, three tables must be maintained. First, the whitelist, $W$ is expected to grow linearly with the number of users. At any given time, $W$ contains a list of \{source IP address, username\} pairs that have been successfully authenticated in the last $t_{1}$ units of time. Second, the number of entries in $F T$ increases by one whenever a remote host makes a failed login attempt using a valid username, if the username is not already in $F T$, and the remote host's IP address is not in $W$ (or has no valid cookie). Therefore, unlike the VS protocol, the total number of valid usernames in the login server puts an upper bound on the number of entries in $F T$ since a failed login attempt for a nonexisting username does not affect this table.

A new entry is added to $F S$ only when a valid \{username, password\} pair is provided from an IP address not used before for this username. Therefore, the number of entries in $F S$ is proportional to the number of IP addresses legitimate users successfully authenticated from. Increasing $t_{3}$ increases the number of entries in $F S$ since the table entries last longer. The number of entries in $F S$ is expected to be close to the number of active users within the last $t_{3}$ units of time (as also shown in the analysis of two realworld data sets in Section 5).

\subsection{Limitations}

Table 3 summarizes major shortcomings in the PS, VS, and PGRP protocols. Under each protocol, the text is highlighted in bold face if the corresponding entry is a limitation. The first limitation in the security row represents Q1 as discussed in Section 4.1.1. The second limitation is about password space elimination for an adversary with a valid cookie or who can use an IP address from which a username has been successfully authenticated in the past. Neither the PS nor VS protocol restricts the number of failed login attempts for such adversaries. Cookie theft is a possible attack that can be mounted against all these protocols, but can be mitigated by updating a counter in the cookie for the maximum number of failed login attempts [17]. The VS protocol stores cookies only on trustworthy machines (as discussed in Section 6), to reduce exposure to cookie theft.

As outlined in Table 3, the first limitation in the usability row is about subsequent login from a known machine, as discussed in Section 4.2. Only PGRP allows legitimate users to try a relatively large number of wrong passwords $\left(k_{1}=30\right.$ default) without passing ATTs. In the second usability limitation, only the VS protocol allows an adversary to make enough failed login attempts for the valid usernames so that legitimate users must then pass ATTs first. The third usability limitation is about ATT challenges for a user who successfully logs in from an unknown machine for the first time (as discussed in Section 4.2). Usability drawbacks of cookies are discussed in Section 3.3. By using either IP addresses or both cookies and IP addresses for tracking legitimate users, PGRP is the only protocol that avoids usability drawbacks of using cookies.

As discussed in Section 2, the design of the deterministic function AskATT() in both PS and VS protocols could have security and deployability drawbacks. Given that the design of both PS and VS protocols considers only cookies to identify machines, only PGRP is designed for both login systems that are web-based and those that are not webbased (e.g., SSH and FTP). The last two limitations in the deployability row are as discussed in Section 4.3.

\section{Empirical Evaluation}

In this section, we provide the details of our test setup, empirical results, and analysis of PGRP on two different data sets. PGRP results are also compared to those obtained from testing the PS and VS protocols on the same data sets.

\subsection{Data Sets}

We used two data sets from an operational university network environment. Each data set logs events of a particular remote login service, over a one-year period each.

SSH Server Log. The first data set was a log file for an SSH server serving about 44 user accounts. The SSH server recorded details of each authentication event, including: date, time, authentication status (success, failed, or invalid username), username, source IP address, and source port. Log files were for the period of January 4, 2009 to January 22, 2010 (thus, slightly over one year). Table 4 shows that the majority of the login events (95 percent) are for invalid usernames suggesting that most login attempts are due to SSH guessing attacks. Note that attack login attempts 
TABLE 4

Login Events from SSH (Jan. 4, 2009 to Jan. 22, 2010) and Horde Email Servers (Jan. 15, 2009 to Jan. 25, 2010)

\begin{tabular}{|c|r|r|}
\hline Number of: & SSH log & Email log \\
\hline \hline a) Login events & 90,190 & 48,375 \\
i) with valid usernames & $5 \%$ & $99 \%$ \\
ii) with invalid usernames & $95 \%$ & $1 \%$ \\
b) Valid usernames entered & 26 & 147 \\
c) Invalid usernames entered & 13,654 & 130 \\
\hline
\end{tabular}

involving valid usernames are not distinguishable from incorrect logins by legitimate users since there is no indication whether the source is malicious or benign. However, there were only few failed login attempts for valid usernames either over short bursts or over the whole log capture period. The number of invalid usernames that appear to be mistyped valid usernames represents less than one percent.

Email server log (web interface). The second data set consisted of $\log$ files of a Horde IMP email client ${ }^{2}$ for the period of January 15, 2009 to January 25, 2010. The Horde email platform is connected to an IMAP email server in a university environment. For each authentication event, a log entry contained: date, time, authentication status (success, failed, or invalid username), username, and source IP address. Although the number of registered user accounts in this server is 1,758, only 147 accounts were accessed. Compared to the SSH log, Table 4 shows that malicious login attempts are far less prevalent, at only about one percent. Login attempts with valid usernames generated by guessing attacks are, as above, not distinguishable. We were unable to determine the percentage of misspelled valid usernames since the log file data including the usernames was anonymized.

\subsection{Simulation Method and Assumptions}

We performed a series of experiments with a Python-based implementation of PGRP with different settings of the configuration variables $\left(k_{1}, k_{2}, t_{1}, t_{2}\right.$, and $\left.t_{3}\right)$. The login events in each data set are ordered according to date (older entries first). Each event is processed by PGRP as if it runs in real time, with protocol tables updated according to the events. Since entries in the tables $W, F T$, and $F S$ have write-expiry intervals, ${ }^{3}$ they get updated at each login event according to the date/time of the current event (i.e., the current time of the protocol is the time of the login event being processed).

We assume that users always answer ATT challenges correctly. While some users will fail in answering some ATTs in practice (see, e.g., [3]), the percentage of failed ATTs depends on the mechanism used to generate the ATTs, the chosen challenge degree of difficulty (if configurable), and the type of the service and its users. The number of generated ATTs by the server can be updated accordingly; for example, if the probability of answering an ATT correctly is $p$, then the total number of generated ATTs must be multiplied by a factor of $1 / p$. Since no browser cookie mechanism was implemented in our tests, in either services of the data sets, the function Valid (cookie, un, $k_{1}$, status) always returns

2. Horde IMP is an open source PHP-based Webmail client for IMAP; see http:/ / www.horde.org/imp/.

3. For an explanation of the use of expiry intervals, see Section 3.2 under "Data structures." false. In the absence of a browser cookie mechanism, a machine from which a user has previously logged in successfully would not be identified by the login system if the machine uses a different IP address that is not in $W$ (see Section 3.3 for further discussion). Such legitimate users will be challenged with ATTs in this case.

For a comparative analysis, we also implemented the PS and VS protocols under the same assumptions. The cookie mechanism in these protocols is replaced by IP address tracking of user machines since cookies are not used in either data sets. The probability $p$ of the deterministic function (see Section 6) is set to 0.05 (suggested by Pinkas and Sander [17]), 0.30 , and 0.60 in each experiment. For VS, $b_{1}$ and $b_{2}$ (see Section 6) are both set to 5 (van Oorschot and Stubblebine [23] suggested 10 as an upper bound for both $b_{1}$ and $b_{2}$ ).

\subsection{Analysis of Results}

In Table 5, we list the protocol parameter settings of eight experiments. For both SSH and email data sets, the total number of ATTs that would be served over the log period, and the maximum number of entries in the $W, F T$, and $F S$ tables are reported.

In the first five experiments, we change the parameter $k_{2}$ from 0 to 4 . $k_{2}$ bounds the number of failed login attempts after which an ATT challenge will be triggered for the following login attempt. Note that the total number of ATTs served over the log period decreases slightly with a larger $k_{2}$ for both data sets. Other parameters have minor effects on the number of ATTs served.

The number of entries in $W$ in the email data set is larger than the SSH data set since there are more email users. Note that although the number of failed login attempts is larger in the SSH data set, the number of entries in FT is smaller than the email data set because the number of usernames is less in the SSH data set with very few common usernames (e.g., common first or last names that can be used in brute force attacks). Given that the protocol requires an ATT for each failed login attempt from a source not in $W$ (and with no valid cookie) when $k_{2}$ is set to 0 , the $F T$ table is empty in the first experiment for both data sets (as the second condition in line 19 in Fig. 1 is always false). Increasing $t_{3}$ increases the number of entries in $F S$ since the table entries last longer as in the seventh experiment.

Tables 6 and 7 show the results of the PS, VS, and PGRP protocols for the SSH and email data sets, respectively. Configuration variables not listed in the settings columns for PGRP are the default values (as in Fig. 1). Test results are analyzed from different perspectives below.

a. The number of successful login attempts. The larger the ratio of successful login attempts without answering ATTs to total successful login attempts, the more convenient the login experience for the user. For the default parameters of PGRP (i.e., $k_{2}=3$ in Tables 6 and 7 and other parameters as given in Fig. 1), the ratio is $4,166 /(4,166+3)=0.999$ for the SSH data set and $46,201 /(46,201+26)=0.999$ for the email data set. The ratio decreases slightly as $k_{2}$ is decreased in both data sets. No other parameters significantly affect this ratio. All the experiments have a ratio over 99 percent except when $k_{2}$ is 0 for the email data set (89 percent). Both PS and VS protocols have a ratio of $3823 /(3823+346)=91 \%$ for the SSH data set and 90 percent for the email data set.

b. The number of unique usernames in successful logins. For PGRP default parameters, the number of unique usernames in successful logins that involved answering 
TABLE 5

Number of ATTs Triggered and Number of Entries in $W, F T$, and $F S$ for PGRP (Nondefault Parameters Are Shaded; for Each Experiment, Changes in Results from the Previous Experiment Are in Bold Face)

\begin{tabular}{|c|c|c|c|c|c|c|c|c|c|c|c|c|c|}
\hline \multirow{2}{*}{$\begin{array}{c}\text { Exp. } \\
\text { no. }\end{array}$} & \multicolumn{5}{|c|}{ Protocol Settings } & \multicolumn{2}{|c|}{ Number of ATTs } & \multicolumn{2}{|c|}{ Entries in $W$} & \multicolumn{2}{|c|}{ Entries in FT } & \multicolumn{2}{|c|}{ Entries in FS } \\
\hline & $k_{1}$ & $k_{2}$ & $t_{1}$ & $t_{2}$ & $t_{3}$ & $\mathrm{SSH}$ & Email & $\mathrm{SSH}$ & Email & $\mathrm{SSH}$ & Email & $\mathrm{SSH}$ & Email \\
\hline 1 & 30 & 0 & 30 & 1 & 1 & 86,118 & 6,232 & 70 & 524 & 0 & 0 & 12 & 56 \\
\hline 2 & 30 & 1 & 30 & 1 & 1 & 85,669 & 1,002 & 70 & 524 & 1 & 1 & 12 & 56 \\
\hline 3 & 30 & 2 & 30 & 1 & 1 & 85,592 & 728 & 70 & 524 & 6 & 9 & 12 & 56 \\
\hline 4 & 30 & 3 & 30 & 1 & 1 & 85,552 & 646 & 70 & 524 & 6 & 9 & 12 & 56 \\
\hline 5 & 30 & 4 & 30 & 1 & 1 & 85,540 & 617 & 70 & 524 & 6 & 9 & 12 & 56 \\
\hline 6 & 10 & 3 & 30 & 1 & 1 & 85,552 & 668 & 70 & 524 & 6 & 9 & 12 & 56 \\
\hline 7 & 30 & 3 & 30 & 2 & 2 & 85,554 & 656 & 70 & 524 & 6 & 9 & 16 & 79 \\
\hline 8 & 30 & 3 & 10 & 1 & 1 & 85,552 & 678 & 41 & 219 & 6 & 9 & 12 & 56 \\
\hline
\end{tabular}

TABLE 6

Experimental Results for the SSH Data Set (Best Results Are Shaded)

\begin{tabular}{|c|c|c|c|c|c|c|c|c|c|c|c|}
\hline \multirow[b]{3}{*}{ Protocol } & \multirow[b]{3}{*}{ Settings } & \multicolumn{4}{|c|}{$\begin{array}{l}\text { Successful Login } \\
\text { number of: }\end{array}$} & \multicolumn{6}{|c|}{$\begin{array}{l}\text { Failed Login } \\
\text { number of: }\end{array}$} \\
\hline & & \multicolumn{2}{|c|}{ a) attempts } & \multicolumn{2}{|c|}{$\begin{array}{l}\text { b) unique } \\
\text { usernames }\end{array}$} & \multicolumn{2}{|c|}{$\begin{array}{l}\text { c) attempts using } \\
\text { valid usernames }\end{array}$} & \multicolumn{2}{|c|}{$\begin{array}{l}\text { d) unique valid } \\
\text { usernames }\end{array}$} & \multicolumn{2}{|c|}{$\begin{array}{l}\text { e) attempts using } \\
\text { invalid usernames }\end{array}$} \\
\hline & & $\begin{array}{l}\mathrm{w} / \\
\text { ATT }\end{array}$ & $\begin{array}{l}\text { w/o } \\
\text { ATT }\end{array}$ & $\begin{array}{l}\text { w/ } \\
\text { ATT }\end{array}$ & $\begin{array}{l}\text { w/o } \\
\text { ATT }\end{array}$ & $\begin{array}{l}\mathrm{w} / \\
\text { ATT }\end{array}$ & $\begin{array}{l}\text { w/o } \\
\text { ATT }\end{array}$ & $\begin{array}{l}\text { w/ } \\
\text { ATT }\end{array}$ & $\begin{array}{l}\text { w/o } \\
\text { ATT }\end{array}$ & $\begin{array}{l}\text { w/ } \\
\text { ATT }\end{array}$ & $\begin{array}{l}\text { w/o } \\
\text { ATT }\end{array}$ \\
\hline \multirow{3}{*}{ PS [17] } & $p=0.05$ & \multirow{3}{*}{346} & \multirow{3}{*}{3,823} & \multirow{3}{*}{24} & \multirow{3}{*}{23} & 0 & 563 & 0 & 20 & 3,930 & 81,528 \\
\hline & $p=0.30$ & & & & & 146 & 417 & 4 & 16 & 19,015 & 66,443 \\
\hline & $p=0.60$ & & & & & 557 & 6 & 18 & 2 & 79,408 & 6,050 \\
\hline \multirow{3}{*}{ VS [23] } & $p=0.05$ & \multirow{3}{*}{346} & \multirow{3}{*}{3,823} & \multirow{3}{*}{24} & \multirow{3}{*}{23} & 418 & 145 & 12 & 20 & 50,806 & 34,652 \\
\hline & $p=0.30$ & & & & & 444 & 119 & 14 & 16 & 59,543 & 25,915 \\
\hline & $p=0.60$ & & & & & 557 & 6 & 18 & 2 & 82,160 & 3,298 \\
\hline \multirow{8}{*}{ PGRP } & $k_{2}=0$ & 412 & 3,757 & 24 & 23 & 248 & 315 & 16 & 14 & \multirow{8}{*}{85,458} & \multirow{8}{*}{0} \\
\hline & $k_{2}=1$ & 50 & 4,119 & 13 & 24 & 161 & 402 & 13 & 20 & & \\
\hline & $k_{2}=2$ & 20 & 4,149 & 7 & 24 & 114 & 449 & 11 & 20 & & \\
\hline & $k_{2}=3$ & 3 & 4,166 & 3 & 24 & 91 & 472 & 5 & 20 & & \\
\hline & $k_{2}=4$ & 1 & 4,168 & 1 & 24 & 81 & 482 & 3 & 20 & & \\
\hline & $k_{1}=10$ & 3 & 4,166 & 3 & 24 & 91 & 472 & 5 & 20 & & \\
\hline & $t_{2}=2, t_{3}=2$ & 5 & 4,164 & 3 & 24 & 91 & 472 & 5 & 20 & & \\
\hline & $t_{1}=10$ & 3 & 4,166 & 3 & 24 & 91 & 472 & 5 & 20 & & \\
\hline
\end{tabular}

ATTs (in the SSH data set) is three. Thus, the majority of valid users were not challenged with any ATT. For the other data set, 11 valid usernames (out of 147) faced an ATT challenge. Almost all usernames were used in successful logins without answering ATTs in both data sets. $k_{2}$ and $t_{2}$ are the only parameters that affected the results. For both data sets, most SSH users were asked to answer ATTs in both the PS and VS protocols; therefore, PGRP offers a more convenient login for legitimate users.

c. The number of failed login attempts with valid usernames. Failed login attempts with valid usernames could be from either malicious or benign sources. In the first experiment on PGRP $\left(k_{2}=0\right)$, there are 315 failed attempts not involving ATTs in the SSH data set and 1,199 in the email data set. Given that the source IP addresses of all these attempts are in $W$, these failed attempts are considered benign. In general, the lower the number of attempts with ATTs the better for user convenience. For PGRP default parameter settings, 16 percent $(91 /(472+91))$ of the failed attempts (with valid usernames) involved ATT challenges in the SSH data set and three percent $(46 /(1,528+46))$ in the email data set. Even if we assume that all failed attempts (with ATTs) are made by legitimate users, PGRP results are better compared to 74 percent $(418 /(418+145))$ for the SSH data set and 61 percent for the email data set in the VS best case (for $p=0.05$ ). PS offers slightly better results, however, this is only when $p=0.05$ which also reduces the number of required ATTs for password guessing attempts (i.e., with invalid usernames as in the last column in Tables 6 and 7).

d. The number of unique valid usernames in failed login attempts. In both data sets, setting $k_{2} \geq 1$ in PGRP causes a significant decrease in the number of unique valid usernames that face ATT challenges in failed login attempts. Other parameters have no significant effect in this manner. For $k_{2}=3$ (default value), in both data sets the number of affected usernames (i.e., the number of legitimate users that are asked to answer ATTs for failed login attempts) is comparable to PS results but less than VS; therefore, PGRP offers a more convenient login for legitimate users.

e. The number of failed login attempts with invalid usernames. Any login attempt with invalid username triggers an ATT in PGRP (i.e., no failed login attempt with invalid usernames avoids an ATT). Indeed, all attempts with invalid usernames trigger ATTs in both data sets. In contrast, for the SSH data set, only 0.046 percent in PS and 0.59 percent in VS trigger ATTs for $p=0.05$ (0.04 percent and 0.51 percent in the email data set).

Summary of Comparison. The trade-off between user convenience (item 3 above) and login security with respect to password guessing (item 5) in both PS and VS protocols is evident from the above discussion; i.e., increasing the number of ATTs to limit password guessing attempts also increases the number of ATTs legitimate users must answer. Such a trade-off is significantly limited with PGRP. 
TABLE 7

Experimental Results for the Email Data Set (Best Results Are Shaded)

\begin{tabular}{|c|c|c|c|c|c|c|c|c|c|c|c|}
\hline \multirow{2}{*}{ Protocol } & \multirow{2}{*}{ Settings } & \multicolumn{4}{|c|}{$\begin{array}{l}\text { Successful Login } \\
\text { number of: }\end{array}$} & \multicolumn{6}{|c|}{$\begin{array}{l}\text { Failed Login } \\
\text { number of: }\end{array}$} \\
\hline & & \multicolumn{2}{|c|}{ a) attempts } & \multicolumn{2}{|c|}{$\begin{array}{l}\text { b) unique } \\
\text { usernames }\end{array}$} & \multicolumn{2}{|c|}{$\begin{array}{l}\text { c) attempts using } \\
\text { valid usernames }\end{array}$} & \multicolumn{2}{|c|}{$\begin{array}{l}\text { d) unique valid } \\
\text { usernames }\end{array}$} & \multicolumn{2}{|c|}{$\begin{array}{l}\text { e) attempts using } \\
\text { invalid usernames }\end{array}$} \\
\hline \multirow[b]{2}{*}{ PS [17] } & $p=0.05$ & \multirow[b]{2}{*}{4,609} & \multirow[b]{2}{*}{4,1618} & \multirow[b]{2}{*}{134} & \multirow[b]{2}{*}{102} & 166 & 1,408 & 7 & 101 & 24 & 550 \\
\hline & $p=0.30$ & & & & & 442 & 1,132 & 29 & 79 & 85 & 489 \\
\hline \multirow{3}{*}{ VS [23] } & $p=0.05$ & \multirow{3}{*}{4,609} & \multirow{3}{*}{41,618} & \multirow{3}{*}{134} & \multirow{3}{*}{102} & 961 & 613 & 41 & 101 & 291 & 283 \\
\hline & $p=0.30$ & & & & & 1,103 & 471 & 55 & 79 & 350 & 224 \\
\hline & $p=0.60$ & & & & & 1,528 & 46 & 100 & 10 & 545 & 29 \\
\hline \multirow{5}{*}{ PGRP } & $k_{2}=0$ & 5,283 & 40,944 & 134 & 100 & 375 & 1,199 & 88 & 71 & \multirow{5}{*}{574} & \multirow{5}{*}{0} \\
\hline & $k_{2}=1$ & 279 & 45,948 & 69 & 127 & 149 & 1,425 & 47 & 108 & & \\
\hline & $k_{2}=2$ & 81 & 46,146 & 32 & 132 & 73 & 1,501 & 18 & 108 & & \\
\hline & $t_{2}=2, t_{3}=2$ & 36 & 46,191 & 11 & 134 & 46 & 1,528 & 11 & 108 & & \\
\hline & $t_{1}=10$ & 34 & 46,193 & 13 & 134 & 70 & 1,504 & 13 & 108 & & \\
\hline
\end{tabular}

Moreover, the number of legitimate login attempts that trigger ATTs (and the number of affected users) is significantly lower in PGRP than both PS and VS. On the other hand, in PGRP, more ATTs must be answered in password guessing attacks; if $g$ is the number of password guessing attempts for $m$ usernames, PGRP requires answering ATT challenges for at least $g-k_{2} m$ password guessing attempts. Our data sets represent two very different scenarios: the SSH server received almost 95 percent invalid login attempts, and the email server received only one percent of such attempts (see Table 4). Yet, as the above analysis indicates, PGRP is significantly better (for both security and usability) than previous ATT-based protocols in both cases, and it can be deployed without affecting the login experience of legitimate users.

\section{Background on Previous ATt-Based Protocols}

Pinkas and Sander [17] introduced the topic with a strawman login protocol (see pseudocode in Fig. 2) that requires answering an ATT challenge first before entering the \{username, password\} pair. Failing to answer the ATT correctly prevents the user from proceeding further. This protocol requires the adversary to pass an ATT challenge for each password guessing attempt, in order to gain information about correctness of the guess.

While this simple protocol is effective against online dictionary attacks assuming that the used ATTs are secure, legitimate users must also pass an ATT challenge for every login attempt. Therefore, this protocol affects user convenience substantially, and requires the login server to generate an ATT challenge for every login attempt.

Pinkas and Sander [17] then made their actual proposal, a login protocol that reduces the number of ATTs legitimate users are required to pass; see pseudocode in Fig. 3 (PS protocol). The protocol stores a browser cookie on the machine of users who had previously logged in successfully. The cookie is tied to the username of the last successful login attempt.

Once the user requests the login server URL, the user's browser sends the cookie (if any) back to the server. The protocol then requests the user to enter a \{username, password pair. If the pair is correct and a valid cookie (i.e., an unexpired cookie indicating that a successful login for the username was made from the same browser) is received from the browser then the user is granted access. If the pair is correct but no valid cookie is received, then an ATT challenge must be answered before account access is granted. Otherwise, if the \{username, password pair is incorrect then according to a function AskATT (username, password), an ATT challenge might be required before informing the user that the \{username, password\} pair is incorrect.

AskATT(username, password) must be a deterministic function of the entered \{username, password\} pair such that for a specific pair, an ATT challenge is either always requested, or never (this function is denoted AskATT (un, pw) in Fig. 3). That is, for a password space of size $N, p N$ of the possible passwords require ATTs (e.g., if $p=0.05,0.05 \times N$ of the password space for a given username require ATTs).

With this protocol, legitimate users must pass ATTs in the following cases: 1) when the user logs in from a machine for the first time; and 2) when the user's \{username, password\} pair is incorrect and $A s k A T T()$ triggers an ATT. On the other hand, an automated program needs to correctly answer an ATT for each password guessing attempt except one case: when the \{username, password\} pair is incorrect and a deterministic function $\operatorname{AskATT}()$ did not request an ATT.

In addition to the correct password, this protocol requires ATTs for a fraction $p$ of the incorrect passwords. Therefore, an adversary can confirm that $(1-p)(N-1) \approx N-p N$ of the passwords in the password space $N$ are incorrect without answering any ATT challenge. The expected number of ATTs an adversary must correctly answer to guess a password correctly is $\frac{1}{2} p N$. Thus, if the adversary is willing to answer $c$ ATTs, the probability of finding a correct password is $c / p N$. For better defence against online dictionary attacks, the function $A s k A T T()$ should request ATTs for the majority of the possible passwords in the overall password space (e.g., $p>0.75$ ). However, the probability that a legitimate user is given an ATT challenge upon entering an incorrect password will also increase, creating a 
trade-off between password security and user convenience. In fact, setting $p=1$ makes this protocol similar to the strawman protocol, except for successful logins with valid cookies where no ATT is required.

Van Oorschot and Stubblebine [23] proposed modifications to the previous protocol (see Fig. 4; VS protocol) which track failed logins per username to impose ATT challenges after exceeding a configurable threshold of failures (threshold $b_{1}$ for correct \{username, password\} pair and threshold $b_{2}$ for incorrect pair; see Fig. 4). Hence, for an incorrect \{username, password\} pair, the decision to request an ATT not only depends on the function $A s k A T T()$ but also on the number of failed login attempts for the username (line 13 in Fig. 4).

In addition, upon entering correct credentials in the absence of a valid cookie, the user is asked whether the machine in use is trustworthy and if the user uses it regularly. The cookie is stored in the user's machine only if the user responds yes to the question. This approach aims to reduce the possibility of cookie theft since a negative answer is expected if the user logs in from a public machine. The user account is set to be in nonowner mode for a specified time window when a login is successful without receiving a valid cookie from the user machine; otherwise the account is set to owner mode.

The number of incorrect passwords that an adversary can eliminate without passing any ATT challenge is decreased to about $(1-p) b_{2}$. Moreover, the adversary is expected to need to correctly answer about $N / 2$ ATTs in order to guess a password correctly as opposed to $\frac{1}{2} p N$ in the PS protocol. While this VS protocol addresses the security drawback of the PS [17] algorithm, the legitimate user always faces an ATT challenge once the threshold $b_{2}$ is exceeded. This feature enables adversaries to affect user login convenience, by initiating $\geq b_{2}$ failed login attempts for each targeted username, forcing ATT challenges for the subsequent login attempts.

\section{Concluding Remarks}

Online password guessing attacks on password-only systems have been observed for decades (see, e.g., [21]). Presentday attackers targeting such systems are empowered by having control of thousand to million-node botnets. In previous ATT-based login protocols, there exists a securityusability trade-off with respect to the number of free failed login attempts (i.e., with no ATTs) versus user login convenience (e.g., less ATTs and other requirements). In contrast, PGRP is more restrictive against brute force and dictionary attacks while safely allowing a large number of free failed attempts for legitimate users. Our empirical experiments on two data sets (of one-year duration) gathered from operational network environments show that while PGRP is apparently more effective in preventing password guessing attacks (without answering ATT challenges), it also offers more convenient login experience, e.g., fewer ATT challenges for legitimate users even if no cookies are available. However, we reiterate that no user testing of PGRP has been conducted so far.

PGRP appears suitable for organizations of both small and large number of user accounts. The required system resources (e.g., memory space) are linearly proportional to the number of users in a system. PGRP can also be used with remote login services where cookies are not applicable (e.g., SSH and FTP).

\section{ACKNOWLEDGMENTS}

The second author is supported by an NSERC postdoctoral fellowship and by NSERC ISSNet. The third author is Canada Research Chair in Authentication and Computer Security and acknowledges NSERC for funding the chair, and a Discovery Grant. Partial funding from NSERC ISSNet is also acknowledged.

\section{REFERENCES}

[1] Amazon Mechanical Turk. https://www.mturk.com/mturk/, June 2010

[2] S.M. Bellovin, "A Technique for Counting Natted Hosts," Proc. ACM SIGCOMM Workshop Internet Measurement, pp. 267-272, 2002.

[3] E. Bursztein, S. Bethard, J.C. Mitchell, D. Jurafsky, and C. Fabry, "How Good Are Humans at Solving CAPTCHAs? A Large Scale Evaluation," Proc. IEEE Symp. Security and Privacy, May 2010.

[4] M. Casado and M.J. Freedman, "Peering through the Shroud: The Effect of Edge Opacity on Ip-Based Client Identification," Proc. Fourth USENIX Symp. Networked Systems Design and Implementation (NDSS '07), 2007.

[5] S. Chiasson, P.C. van Oorschot, and R. Biddle, "A Usability Study and Critique of Two Password Managers," Proc. USENIX Security Symp., pp. 1-16, 2006.

[6] D. Florencio, C. Herley, and B. Coskun, "Do Strong Web Passwords Accomplish Anything?," Proc. USENIX Workshop Hot Topics in Security (HotSec '07), pp. 1-6, 2007.

[7] K. Fu, E. Sit, K. Smith, and N. Feamster, "Dos and Don'ts of Client Authentication on the Web," Proc. USENIX Security Symp., pp. 251-268, 2001.

[8] P. Hansteen, "Rickrolled? Get Ready for the Hail Mary Cloud!," http: / / bsdly.blogspot.com/2009/11/rickrolled-get-ready-forhail-mary.html, Feb. 2010.

[9] Y. He and Z. Han, "User Authentication with Provable Security against Online Dictionary Attacks," J. Networks, vol. 4, no. 3, pp. 200-207, May 2009.

[10] T. Kohno, A. Broido, and K.C. Claffy, "Remote Physical Device Fingerprinting," Proc. IEEE Symp. Security and Privacy, pp. 211-225, 2005.

[11] M. Motoyama, K. Levchenko, C. Kanich, D. Mccoy, G.M. Voelker, and S. Savage, "Re: CAPTCHAs Understanding CAPTCHASolving Services in an Economic Context," Proc. USENIX Security Symp., Aug. 2010.

[12] C. Namprempre and M.N. Dailey, "Mitigating Dictionary Attacks with Text-Graphics Character Captchas," IEICE Trans. Fundamentals of Electronics, Comm. and Computer Sciences, vol. E90-A, no. 1, pp. 179-186, 2007.

[13] A. Narayanan and V. Shmatikov, "Fast Dictionary Attacks on Human-Memorable Passwords Using Time-Space Tradeoff," Proc. ACM Computer and Comm. Security (CCS '05), pp. 364-372, Nov. 2005.

[14] Nat'1 Inst. of Standards and Technology (NIST), Hashbelt. http:/ / www.itl.nist.gov/div897/sqg/dads/HTML/hashbelt.html, Sept. 2010 .

[15] "The Biggest Cloud on the Planet Is Owned by ... the Crooks," NetworkWorld.com., http://www.networkworld.com/ community/node/58829, Mar. 2010.

[16] J. Nielsen, "Stop Password Masking," http://www.useit.com/ alertbox/passwords.html, June 2009.

[17] B. Pinkas and T. Sander, "Securing Passwords against Dictionary Attacks," Proc. ACM Conf. Computer and Comm. Security (CCS '02) pp. 161-170, Nov. 2002.

[18] D. Ramsbrock, R. Berthier, and M. Cukier, "Profiling Attacker Behavior following SSH Compromises," Proc. 37th Ann. IEEE/IFIP Int'l Conf. Dependable Systems and Networks (DSN '07), pp. 119-124, June 2007. 
[19] SANS.org, "Important Information: Distributed SSH Brute Force Attacks," SANS Internet Storm Center Handler's Diary, http:// isc.sans.edu/diary.html?storyid=9034, June 2010.

[20] "The Top Cyber Security Risks," SANS.org, http://www.sans. org/top-cyber-security-risks/, Sept. 2009.

[21] C. Stoll, The Cuckoo's Egg: Tracking a Spy through the Maze of Computer Espionage. Doubleday, 1989.

[22] "Botnet Pierces Microsoft Live through Audio Captchas," TheRegister.co.uk, http://www.theregister.co.uk/2010/03/22/ microsoft_live_captcha_by pass/, Mar. 2010.

[23] P.C. van Oorschot and S. Stubblebine, "On Countering Online Dictionary Attacks with Login Histories and Humans-in-theLoop," ACM Trans. Information and System Security, vol. 9, no. 3, pp. 235-258, 2006.

[24] L. von Ahn, M. Blum, N. Hopper, and J. Langford, "CAPTCHA: Using Hard AI Problems for Security," Proc. Eurocrypt, pp. 294311, May 2003.

[25] M. Weir, S. Aggarwal, M. Collins, and H. Stern, "Testing Metrics for Password Creation Policies by Attacking Large Sets of Revealed Passwords," Proc. 17th ACM Conf. Computer and Comm. Security, pp. 162-175, 2010.

[26] Y. Xie, F. Yu, K. Achan, E. Gillum, M. Goldszmidt, and T. Wobber, "How Dynamic Are IP Addresses?," SIGCOMM Computer Comm. Rev., vol. 37, no. 4, pp. 301-312, 2007.

[27] J. Yan and A.S.E. Ahmad, "A Low-Cost Attack on a Microsoft CAPTCHA," Proc. ACM Computer and Comm. Security (CCS '08), pp. 543-554, Oct. 2008.

[28] J. Yan and A.S.E. Ahmad, "Usability of CAPTCHAs or Usability Issues in CAPTCHA Design," Proc. Symp. Usable Privacy and Security (SOUPS '08), pp. 44-52, July 2008.

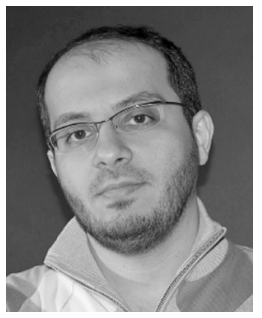

Mansour Alsaleh received the master's degree of computer science at the University of Ottawa in 2006. He is working toward the $\mathrm{PhD}$ degree in computer science at Carleton University. Prior to his master studies, Mansour had four years industry work experience in data security. His main research interests span through network security, scan detection, intrusion detection, network security data visualization, authentication, e-privacy, and identity federation.

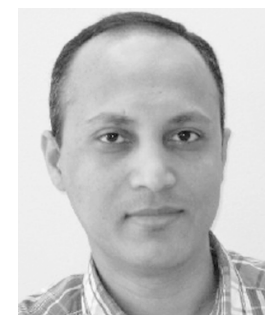

Mohammad Mannan received the PhD degree in Computer Science from Carleton University in 2009 in the area of Internet authentication and usable security. He is an assistant professor at the Concordia Institute for Information Systems Engineering, Concordia University, Montreal. He was a post-doctoral fellow in the Department of Electrical and Computer Engineering at the University of Toronto from 2009 to 2011 (funded by an NSERC PDF and ISSNet). His research interests lie in the area of Internet and systems security, with a focus on solving high-impact security and privacy problems of today's Internet.

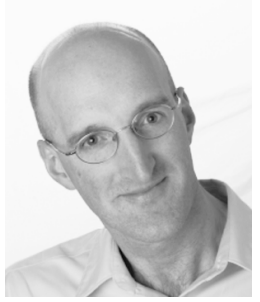

P.C. van Oorschot is a professor of computer science at Carleton University in Ottawa, where he is a Canada research chair in Authentication and Computer Security. He was program chair of USENIX Security 2008, Program cochair of NDSS 2001 and 2002, and coauthor of the Handbook of Applied Cryptography (1996). He is on the editorial board of IEEE TIFS and IEEE TDSC. His current research interests include authentication and identity management, security and usability, software security, and computer security. He is a member of the IEEE.

For more information on this or any other computing topic, please visit our Digital Library at www.computer.org/publications/dlib. 